\title{
Post resuscitation electrocardiogram for coronary angiography indication after out-of-hospital cardiac arrest
}

\author{
Florence Leclercq $^{\mathrm{a}, 1}$, Clément Lonjon ${ }^{\mathrm{b}, 1}$, Grégory Marin ${ }^{\mathrm{c}, 1}$, Mariama Akodad ${ }^{\mathrm{a}, 1}$, François Roubille ${ }^{\mathrm{a}, 1}$, \\ Jean-Christophe Macia ${ }^{\mathrm{a}, 1}$, Luc Cornillet ${ }^{\mathrm{b}, 1}$, Richard Gervasoni ${ }^{\mathrm{a}, 1}$, Laurent Schmutz ${ }^{\mathrm{b}, 1}$, Bertrand Ledermann ${ }^{\mathrm{b}, 1}$, \\ Pascal Colson $^{\mathrm{d}, 1}$, Guillaume Cayla ${ }^{\mathrm{b}, 1}$, Benoit Lattuca ${ }^{\mathrm{b}, *, 1}$ \\ a University of Montpellier, Cardiology department, Arnaud de Villeneuve University Hospital, Montpellier, France \\ b Cardiology Department, Caremeau University Hospital, Montpellier University, Nîmes, France \\ c Department of Epidemiology, Medical Statistics and Public Health, Arnaud de Villeneuve University Hospital, Montpellier, France \\ d Department of Anesthesiology and Critical Care Medicine, Arnaud de Villeneuve Hospital, Montpellier, France.
}

Keywords:

Coronary angiography

Cardiac arrest

Resuscitation

Repolarization disorders

ECG

Mortality

\begin{abstract}
A B S T R A C T
Background: Coronary angiography is the standard of care after Out-of-Hospital Cardiac Arrest (OHCA), but its benefit for patients without persistent ST-segment elevation (STE) remains controversial.

Methods: All patients admitted for coronary angiography after a resuscitated OHCA were consecutively included in this prospective study. Three patient groups were defined according to post-resuscitation ECG: STE or new left bundle branch block (LBBB) (group 1); other ST/T repolarization disorders (group 2) and no repolarisation disorders (group 3). The proportion and predictive factors of an acute coronary lesion, defined by acute coronary occlusion or thrombotic lesion or lesion associated with flow impairment, were evaluated according to different groups as well as thirty-day mortality.

Results: Among 129 consecutive patients: 62 (48.1\%), 30 (23.3\%) and 30 (23.3\%) patients were included in groups 1,2 and 3 respectively. An acute coronary lesion was observed in $43 \%(n=55)$ of patients, mainly in group 1 $(n=44,70.9 \%$ ). Initial coronary TIMI $0 / 1$ flow was more frequently observed in group 1 than in group 2 ( $n=$ $25,40.3 \%$ vs $n=1,3.3 \%$ ) and never in group 3. Chest pain and STE or new LBBB were independently associated with an acute coronary lesion (adj. OR $=7.14$ [1.85-25.00]; $p=0.004$ and adj. OR $=11.10$ [3.70-33.33]; $p<0.001$ respectively). In absence of any repolarization disorders, acute coronary lesion or occlusion were excluded with negative predictive values of $93.3 \%$ and $100 \%$ respectively. The one-month survival rate was $38.8 \%$ and was better in patients among the group 1 compared to those from the 2 other groups ( $n=28,45.2 \%$ vs $n=21,35 \%$, respectively; $p=0.014$ ).

Conclusion: Considering the high negative predictive value of post-resuscitation ECG to exclude acute coronary lesion and occlusion after OHCA, a delayed coronary angiography appears a reliable alternative for patients without repolarization disorders.
\end{abstract}

\footnotetext{
* Corresponding author at: Département de cardiologie, Centre Hospitalier Universitaire Caremeau, Place du Pr Debre, 30029 Nîmes Cedex, France.

E-mail addresses: f-leclercq@chu-montpellier.fr (F. Leclercq), g-marin@chu-montpellier.fr (G. Marin), f-roubille@chu-montpellier.fr (F. Roubille), jc-macia@chu-montpellier.fr (J.-C. Macia), luc.cornillet@chu-nimes.fr (L. Cornillet), r-gervasoni@chu-montpellier.fr (R. Gervasoni), laurent.schmutz@chu-nimes.fr (L. Schmutz), bertrand.ledermann@chu-nimes.fr (B. Ledermann), p-colson@chu-montpellier.fr (P. Colson), guillaume.cayla@chu-nimes.fr (G. Cayla), benoit.lattuca@chu-nimes.fr (B. Lattuca).

1 This author takes responsibility for all aspects of the reliability and freedom from bias of the data presented and their discussed interpretation
}

\section{Introduction}

Out-of-hospital cardiac arrest (OHCA) is a leading cause of death and remains a meaningful issue in clinical cardiology, emergency medicine, and public health due to a very low survival rate of 20 to $30 \%$ [1-6]. Based on limited supportive evidence from different registries and small studies [7-10], international guidelines recommend an invasive management of OHCA during both pre- and in-hospital care including vital-organ support and treatment of the underlying cause of the cardiac arrest [11-13]. However, the cause of cardiac arrest is often unclear immediately after the event, and the lack of a definitive diagnosis can lead to uncertainty regarding the appropriate treatment. Although the most frequent cause of cardiac arrest is ischemic heart disease [14-17], the benefit and the best timing of coronary angiography (CAG) in 
unselected OHCA patients are still debated. If ST-segment elevation myocardial infarction (STEMI) is the cause of OHCA, primary percutaneous coronary intervention (PCI) has demonstrated efficacy and reduction in mortality and may improve circulatory function, reduce infarct size and the recurrence of life-threatening arrhythmias $[10,12]$. However, benefits of a systematic immediate CAG are not clearly defined with conflicting results in patients without ST-segment elevation [6,18-22]. Indeed, CAG remains an invasive procedure, associated to potential adverse effects as bleeding complications, acute renal failure or delayed optimal hemodynamic support.

Evaluation of post-resuscitation ECG could be useful as a triage method in association to patients' clinical characteristics to predict significant acute coronary lesions involved to initial cardiac arrest and limit unnecessary immediate transfer of unstable or frail patients in catheterization laboratory. The main objective of this study was to evaluate association between different post-resuscitation ECG patterns and acute coronary lesion requiring urgent revascularization to guide indication and timing of CAG.

\section{Methods}

\subsection{Study population and patient selection}

All consecutive patients, aged over 18 years, admitted in the catheterization laboratory of the 2 interventional University Hospitals of Montpellier and Nimes (France), after a resuscitated OHCA were prospectively included. Each interventional center offers a $24 \mathrm{~h} / 7$ days service for primary $\mathrm{PCl}$ and specialized intensive care units taking care of post-resuscitation patients with available extracorporeal life supports. The catheterization laboratory can be activated either by the emergency department or the mobile intensive care units from a unique national emergency number (\#15), The use of MICU with onboard emergency physicians results in full 17-lead ECG interpretation, rapid and direct transfer to a PCI center, immediate cardio-pulmonary resuscitation and prehospital therapies administration and rapid activation of the shock team with mobile extracorporeal life support facility for the sickest patients. Patients with an obvious non-cardiac cause of OHCA or admitted for OHCA complicating acute disease diagnosed before the cardiac arrest or for whom a CAG was not planned for medical or ethical implications were excluded. Clinical and laboratory data were obtained from electronic medical and laboratory reports. The protocol was approved by the local ethics committee, institutional regulatory authorities and was conducted according to the principals of the Declaration of Helsinki.

\subsection{Study design and experimental protocol}

Three patient groups were defined according to post-resuscitation 17-lead ECG patterns reviewed by two independent blinded experts:

1/ Group 1: ST segment elevation (STE) or new presumed Left Bundle Branch Block (LBBB). STE was considered significant if present in two contiguous leads with the cut-points: $\geq 0.1 \mathrm{mV}$ in all leads other than leads V2-V3 where the following cut points apply: $\geq 0.2 \mathrm{mV}$ in men $\geq 40$ years; $\geq 0.25 \mathrm{mV}$ in men $<40$ years, or $\geq 0.15 \mathrm{mV}$ in women [23] (Supplemental Fig. A). LBBB was defined as QRS duration $>120 \mathrm{~ms}$ with QS or $\mathrm{rS}$ pattern in V1 and broad R waves in lead L, V5 and V6 [24].

2/Group 2: Other repolarization disorders including ST segment depression or/and T waves changes. ST segment depression was considered significant if $\geq 0.05 \mathrm{mV}$ in two contiguous leads [23]. T waves changes were defined by $\mathrm{T}$ inversion $\geq 0.1 \mathrm{mV}$ in two contiguous leads [23] (Supplemental Fig. B).

3/Group 3: No repolarization disorders including normal ECG, Right Bundle Branch Block (RBBB) without other significant changes, lone atrioventricular block, supraventricular arrhythmia without other significant changes. RBBB was defined as QRS duration $>120 \mathrm{~ms}$ with rSR' complex in V1 V2 and S wave in lead L and V5 V6 (Supplemental Fig. C).

\subsection{Study endpoints}

The primary endpoint was the presence of an acute coronary lesion described in post-OHCA coronary angiography according to each group. Acute coronary lesion was defined by an acute coronary occlusion (Thrombolysis In Myocardial Infarction (TIMI) flow grade 0/1) and/or intracoronary thrombus and/or critical coronary stenosis ( $>90 \%$ of lumen diameter) or severe coronary stenosis ( $>70 \%$ of lumen diameter) with TIMI flow impairment [25]. All coronary angiographies were reviewed by 2 independent blinded experts. In case of discrepancy, a third expert opinion was requested.

Secondary end points evaluated all-cause mortality during hospitalization and at 30-day follow-up as well as independent correlates associated with 30-day survival and the presence of an acute coronary lesion.

\subsection{Statistical analysis}

Descriptive analyses used a Shapiro-Wilk tests to assess normality of the distributions, and the quantitative variables were expressed both as mean \pm standard deviation and median (interquartile range), whereas categorical variables were expressed as number of subjects (percentage). Baseline data of the 3 groups were compared using Fisher test and one-way analysis of variances (ANOVA). Survival analyses were carried out using Kaplan-Meyer curves, alongside Log-rank or Wilcoxon $p$-value in order to compare the patient's groups on one hand and the diagnostic of acute coronary lesion on the other hand. Multivariate analyses using a stepwise selection of variables in a logistic regression model and including variables which p-value $<0.20$ in univariate analysis, were performed to evaluate independent correlates of, first, the presence of acute coronary lesion, and then, 30-day mortality. Sensitivity, specificity, negative predictive value and positive predictive value for the diagnostic of an acute coronary lesion were measured considering each patient groups. All tests were 2-sided with a significance level fixed at 5\%. Statistical tests were performed with R 3.1.1 (The R Foundation for Statistical Computing) and SAS 9.2 (SAS Institute Inc., Cary, NC).

\section{Results}

\subsection{Patient characteristics}

From January 2014 to May 2015, 129 consecutive patients were admitted in the catheterization laboratory after a resuscitated OHCA. Among them, $48.1 \%(n=62)$ of patients presented a STE or new presumed LBBB (group 1), 23.3\% $(n=30)$ of patients had other repolarization disorders, $23.3 \%(\mathrm{n}=30)$ of patients had no significant repolarization disorders and ECG classification was not available for $5.4 \%(n=7)$ of patients (Fig. 1$)$. The population included a majority of men $(79.8 \%, n=103$ ) with a mean age of 60.9 years and with initial shockable rhythm among $38.3 \%(n=46)$ of patients. A cardiopulmonary resuscitation (CPR) was started by witness among $58.2 \%$ ( $n=$ 71) of patients. Baseline clinical characteristics were well-balanced between the 3 groups (Table 1 ). OHCA was mostly related to acute coronary lesion ( $43 \%, n=55)$ and neurologic disorders (6\%, $n=8$ ) (Fig. 2).

\subsection{Coronary status}

Normal coronary arteries were observed in $22.5 \%$ of the patients while a multivessel disease was reported in $42.6 \%$ of the patients. No patient had chronic total occlusion. An acute coronary lesion was mainly observed among patients with STE or LBBB $(70.9 \%, n=44)$ and in only 2 patients $(6.7 \%)$ without significant repolarization disorders 


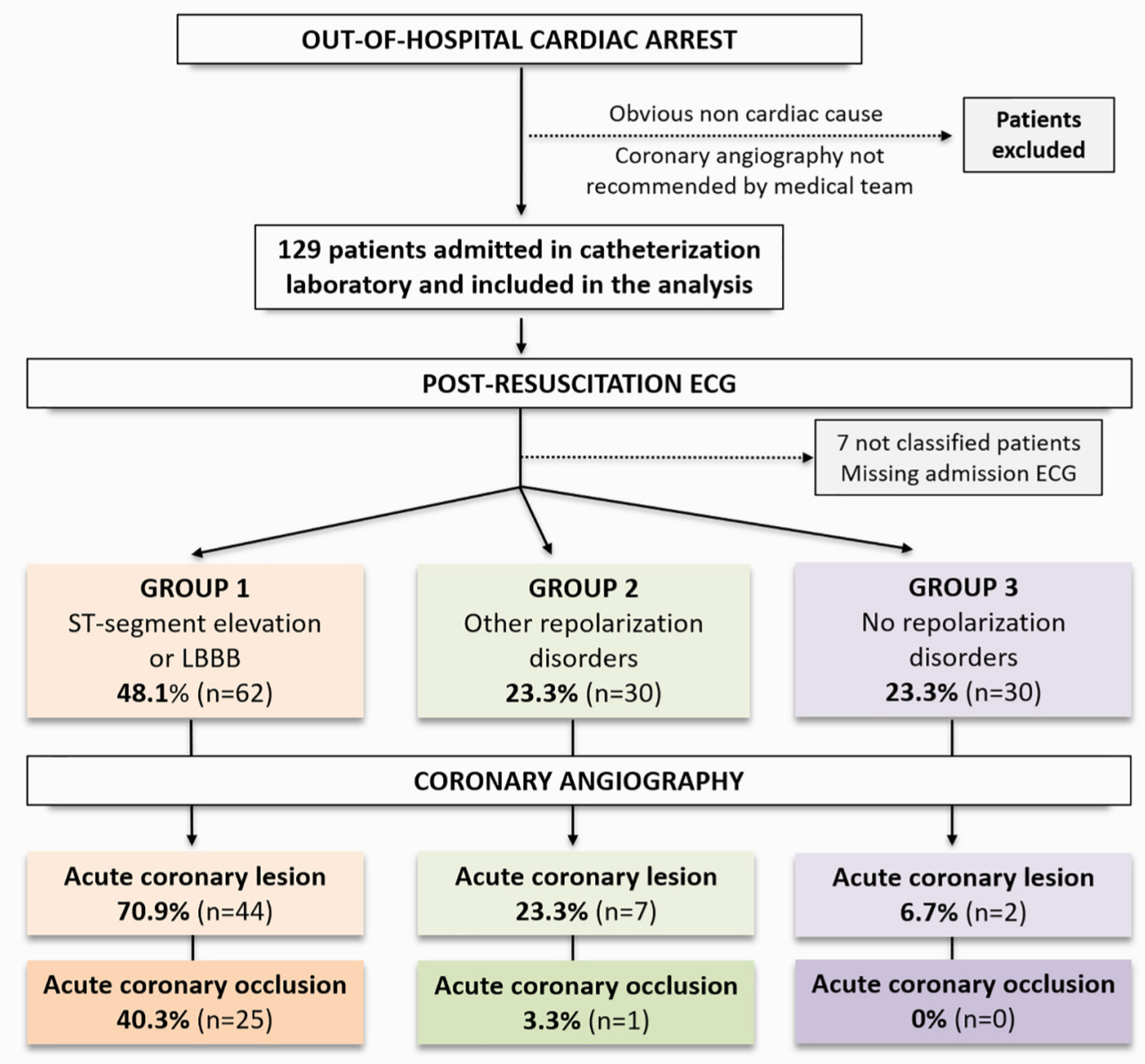

Fig. 1. Study flow chart.

(Table 2). TIMI grade $0 / 1$ flow was more frequent among patients with STE or LBBB (group 1) than those with other repolarization disorders (group 2) (40.3\% vs. 3.3\% respectively). TIMI grade 0/1 was not observed among patients without repolarization disorders (group 3). A PCI was performed immediately in the majority of the patients with an acute

Table 1

Baseline characteristics of the study population.

\begin{tabular}{|c|c|c|c|c|c|}
\hline Variables & $\begin{array}{l}\text { Total population } \\
n=129^{*}\end{array}$ & $\begin{array}{l}\text { Group1: ST-segment elevation } \\
\text { or LBBB } \\
n=62\end{array}$ & $\begin{array}{l}\text { Group 2: Other repolarization } \\
\text { disorders } \\
n=30\end{array}$ & $\begin{array}{l}\text { Group 3: no repolarization } \\
\text { disorders } \\
n=30\end{array}$ & p-value \\
\hline Age (years) & $60.9 \pm 13.8$ & $60.5 \pm 11.1$ & $70.0 \pm 16.6$ & $60.3 \pm 16.3$ & 0.87 \\
\hline Male sex & $103(79.8 \%)$ & $53(85.5 \%)$ & $21(70.0 \%)$ & $25(83.3 \%)$ & 0.19 \\
\hline High blood pressure & $50(38.8 \%)$ & $24(38.7 \%)$ & $14(46.6 \%)$ & $12(40.0 \%)$ & 0.78 \\
\hline Diabetes & $24(18.6 \%)$ & $12(19.3 \%)$ & $6(20.0 \%)$ & $6(20.0 \%)$ & 0.99 \\
\hline Dyslipidemia & $38(29.5 \%)$ & $18(29.0 \%)$ & $11(36.7 \%)$ & $9(30.0 \%)$ & 0.60 \\
\hline Active smoking & $64(49.6 \%)$ & $34(58.8 \%)$ & $13(43.3 \%)$ & $15(50.0 \%)$ & 0.55 \\
\hline Body mass index & $26.3 \pm 5.2$ & $26.9 \pm 5.4$ & $25.8 \pm 4.0$ & $26.1 \pm 6.1$ & 0.73 \\
\hline History of coronary artery disease & $32(24.8 \%)$ & $16(25.8 \%)$ & $8(26.7 \%)$ & $5(16.7 \%)$ & 0.56 \\
\hline Chronic respiratory failure & $17(13.2 \%)$ & $5(8.1 \%)$ & $5(16.7 \%)$ & $6(20.0 \%)$ & 0.25 \\
\hline Initial chest pain & $32(24.8 \%)$ & $26(41.9 \%)$ & $3(10.0 \%)$ & $2(6.7 \%)$ & $<0.01$ \\
\hline Initial dyspnea & $21(16.3 \%)$ & $7(11.3 \%)$ & $6(20.0 \%)$ & $7(23.3 \%)$ & 0.34 \\
\hline Initial shockable rhythm & $74(57.4 \%)$ & $47(75.8 \%)$ & $10(33.3 \%)$ & $12(40.0 \%)$ & $<0.01$ \\
\hline \multirow[t]{2}{*}{ Location of OHCA at home public area } & $55(42.6 \%)$ & $22(35.5 \%)$ & $15(50 \%)$ & $13(43.3 \%)$ & 0.39 \\
\hline & $74(57.4 \%)$ & $40(64.5 \%)$ & $15(50 \%)$ & $17(56.7 \%)$ & \\
\hline Mean duration of no flow (min) & $3.3 \pm 4.3$ & $2.8 \pm 4.3$ & $3.5 \pm 4.1$ & $4.4 \pm 4.6$ & 0.13 \\
\hline Mean duration of low flow (min) & $23.9 \pm 23.3$ & $20.2 \pm 19.2$ & $21.5 \pm 19.4$ & $26.7 \pm 23.2$ & 0.12 \\
\hline Mean time to ROSC ( $\mathrm{min}$ ) & $25.1 \pm 2.6$ & $21.9 \pm 3.4$ & $20.8 \pm 4.6$ & $24.5 \pm 4.7$ & 0.72 \\
\hline CPR by witness & $71(55.0 \%)$ & $31(55.0 \%)$ & $16(53.3 \%)$ & $19(63.3 \%)$ & 0.71 \\
\hline Mean Adrenalin use (mg) & $5.0 \pm 5.4$ & $6.1 \pm 6.6$ & $3.2 \pm 3.2$ & $4.9 \pm 3.9$ & 0.16 \\
\hline $\begin{array}{l}\text { Delay between OHCA and Cardiac } \\
\text { Catheterization (min) }\end{array}$ & $161 \pm 71$ & $167 \pm 71$ & $136 \pm 77$ & $160 \pm 75$ & 0.56 \\
\hline
\end{tabular}

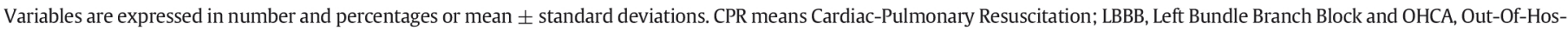
pital Cardiac arrest, ROSC, Return of Spontaneous Circulation. *ECG classification was not available for 7 patients. 


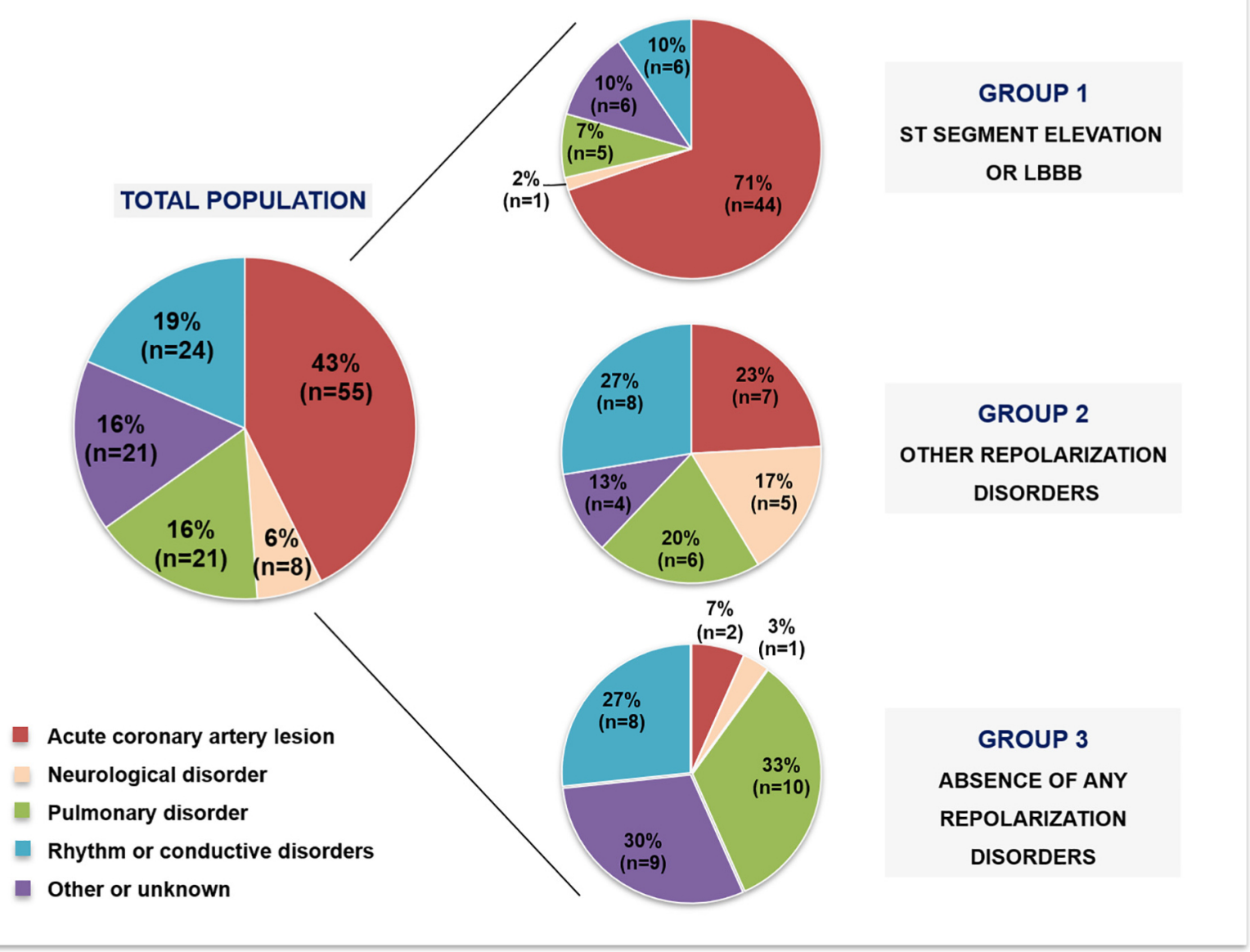

Fig. 2. Distribution of out-of-hospital cardiac arrest etiologies according to post-resuscitation ECG groups.

coronary lesion ( $n=51 / 55 ; 92.7 \%$ ). Among them, only PCI of the acute coronary lesion was mainly performed $(n=45 / 51 ; 88.2 \%)$.

\subsection{Predictive factors of acute coronary lesion}

Chest pain before cardiac arrest and STE or new presumed LBBB on post-resuscitation ECG were independently associated with an acute coronary lesion (adjusted $\mathrm{OR}=7.14[1.85-25.00] ; p=0.004$ and adjusted OR $=11.10$ [3.70-33.33]; $p<0.001$, respectively) (Table 3 ).

The positive and negative predictive values of STE or new presumed LBBB for an acute coronary lesion were $72.1 \%$ (95\%CI [60.9-83.4]) and $85.0 \%$ (95\%CI [75.9-94.1]), respectively, with a sensitivity of $83.0 \%$ (95\%CI [72.9-93.1]) and a specificity of 75.0\% (95\%CI [64.7-85.3]). The sensitivity for diagnostic of acute coronary lesion was largely improved by combining the groups 1 and 2 (all repolarization disorders): $96.2 \%$

Table 2

Baseline angiographic data.

\begin{tabular}{|c|c|c|c|c|c|}
\hline & $\begin{array}{l}\text { Total } \\
\text { population } \\
n=129^{*}\end{array}$ & $\begin{array}{l}\text { Group 1: ST-segment elevation or } \\
\text { LBBB } \\
n=62\end{array}$ & $\begin{array}{l}\text { Group 2: other repolarization } \\
\text { disorders } \\
n=30\end{array}$ & $\begin{array}{l}\text { Group 3: no repolarization } \\
\text { disorders } \\
n=30\end{array}$ & p-value \\
\hline Radial access & $76(58.9 \%)$ & $39(62.9 \%)$ & $13(43.3 \%)$ & $20(66.7 \%)$ & 0.61 \\
\hline Normal coronary arteries & $29(22.5 \%)$ & $3(4.8 \%)$ & $9(30.0 \%)$ & $16(53.3 \%)$ & 0.23 \\
\hline Not-significant coronary disease & $9(7.0 \%)$ & $3(4.8 \%)$ & $2(6.7 \%)$ & $4(13.3 \%)$ & 0.34 \\
\hline Single-vessel coronary disease & $35(27.1 \%)$ & $21(33.9 \%)$ & $5(16.7 \%)$ & $5(16.7 \%)$ & 0.10 \\
\hline Two-vessel coronary disease & $25(19.4 \%)$ & $18(29.0 \%)$ & $4(13.3 \%)$ & $3(10.0 \%)$ & 0.78 \\
\hline Three-vessel coronary disease & $30(23.2 \%)$ & $16(25.8 \%)$ & $10(33.3 \%)$ & $2(6.7 \%)$ & 0.84 \\
\hline Acute coronary lesion & $55(42.6 \%)$ & $44(70.9 \%)$ & $7(23.3 \%)$ & $2(6.7 \%)$ & $<0.01$ \\
\hline Site of acute coronary lesion & & & & & 0.76 \\
\hline Right coronary artery & $20(15.5 \%)$ & $16(25.8 \%)$ & $2(6.7 \%)$ & $1(3.3 \%)$ & \\
\hline Left anterior descending artery & $23(17.8 \%)$ & $18(29.0 \%)$ & $4(13.3 \%)$ & $1(3.3 \%)$ & \\
\hline Circumflex & $8(6.2 \%)$ & $8(12.9 \%)$ & $0(0 \%)$ & $0(0 \%)$ & \\
\hline Left main & $4(3.1 \%)$ & $2(3.2 \%)$ & $1(3.3 \%)$ & $0(0 \%)$ & \\
\hline $\begin{array}{l}\text { Percutaneous coronary } \\
\text { intervention }\end{array}$ & $61(47.3 \%)$ & $47(75.8 \%)$ & $8(26.7 \%)$ & $3(10.0 \%)$ & $<0.01$ \\
\hline Circulatory support system & $9(6.9 \%)$ & $6(9.7 \%)$ & $2(6.7 \%)$ & $0(0 \%)$ & 0.28 \\
\hline Mean contrast volume (mL) & $98.6 \pm 43.6$ & $113.2 \pm 45.8$ & $86.7 \pm 38.8$ & $76.0 \pm 36.1$ & 0.57 \\
\hline
\end{tabular}

Variables are expressed in number and percentages or mean \pm standard deviations. LBBB means Left Bundle Branch Block. ${ }^{*}$ ECG classification was not available for 7 patients. 
Table 3

Independent correlates associated with the presence of an acute coronary lesion.

\begin{tabular}{|c|c|c|c|c|c|c|}
\hline \multirow[t]{2}{*}{ Variables } & \multicolumn{3}{|c|}{ Univariate analysis } & \multicolumn{3}{|c|}{ Multivariate analysis } \\
\hline & OR & $95 \% \mathrm{CI}$ & $p$-value & OR & $95 \% \mathrm{CI}$ & $p$-value \\
\hline Group 1 vs Group 2 and 3 & 14.29 & $33.30-100.00$ & $<0.001$ & 11.10 & $3.70-33.33$ & $<0.001$ \\
\hline Group 1 vs Group 3 & 33.33 & $7.69-100.00$ & $<0.001$ & - & - & - \\
\hline Group 2 vs Group 3 & 4.17 & $0.81-25.00$ & 0.088 & & & \\
\hline Male sex & 1.23 & $0.48-3.13$ & 0.664 & & & \\
\hline Active smoking & 1.45 & $0.70-3.03$ & 0.315 & & & \\
\hline Diabetes & 0.74 & $0.29-1.85$ & 0.513 & & & \\
\hline High blood pressure & 1.04 & $0.50-2.13$ & 0.917 & & & \\
\hline History of CAD & 0.87 & $0.38-1.96$ & 0.743 & & & \\
\hline Chronic respiratory failure & 0.14 & $0.03-0.64$ & 0.011 & 0.057 & $0.005-0.595$ & 0.017 \\
\hline Chest pain before OHCA & 11.11 & $4.00-33.33$ & $<0.001$ & 7.14 & $1.85-25.00$ & 0.004 \\
\hline Initial shockable rhythm & 5.88 & $2.56-14.29$ & $<0.001$ & - & - & - \\
\hline Age* & 0.98 & $0.95-1.01$ & 0.158 & & & \\
\hline Body Mass Index* & 1.04 & $0.96-1.12$ & 0.338 & & & \\
\hline
\end{tabular}

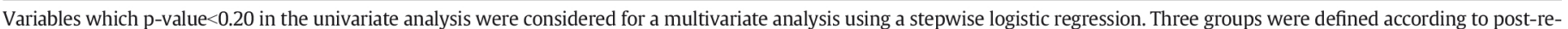

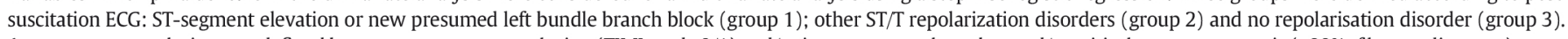

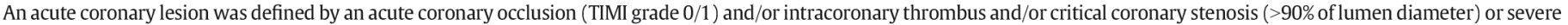
coronary stenosis ( $>70 \%$ of lumen diameter) with TIMI flow impairment.

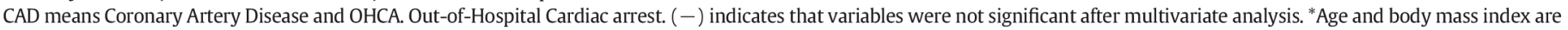
expressed as continuous variables.

(95\%Cl [87.0-99.5]). The absence of significant repolarization disorders on post-resuscitation ECG was associated with a negative predictive value of $93.3 \%$ (95\%CI [84.4-100.0]) for an acute coronary lesion and $100 \%$ considering only acute coronary occlusion.

\subsection{Survival and clinical outcomes}

The in-hospital survival rate was $41.1 \%$ and the 30 -day survival rate was $38.8 \%$. At 30 days, survival was better for patients among the group
1 compared to those from the 2 other groups ( $n=28$ (45.2\%) vs $n=21$ (35\%), respectively; $p=0.014$ ) (Fig. 3A). Similarly, survival was better in case of OHCA related to an acute coronary lesion than to another cause ( $n=27$ (49.1\%) vs $n=23$ (31.5\%), respectively; $p=0.002$ ) (Fig. 3B). After multivariate adjustment (Supplemental Table A), the only positive independent predictive factor of 30-day survival was CPR by witness (adjusted OR 5.55 [1.64-20.00]; $p=0.006$ ) while increasing lactatemia at admission was independently associated with a lower 30-day survival (adjusted OR 0.64 [0.50-0.83]; $p<0.001$ ).

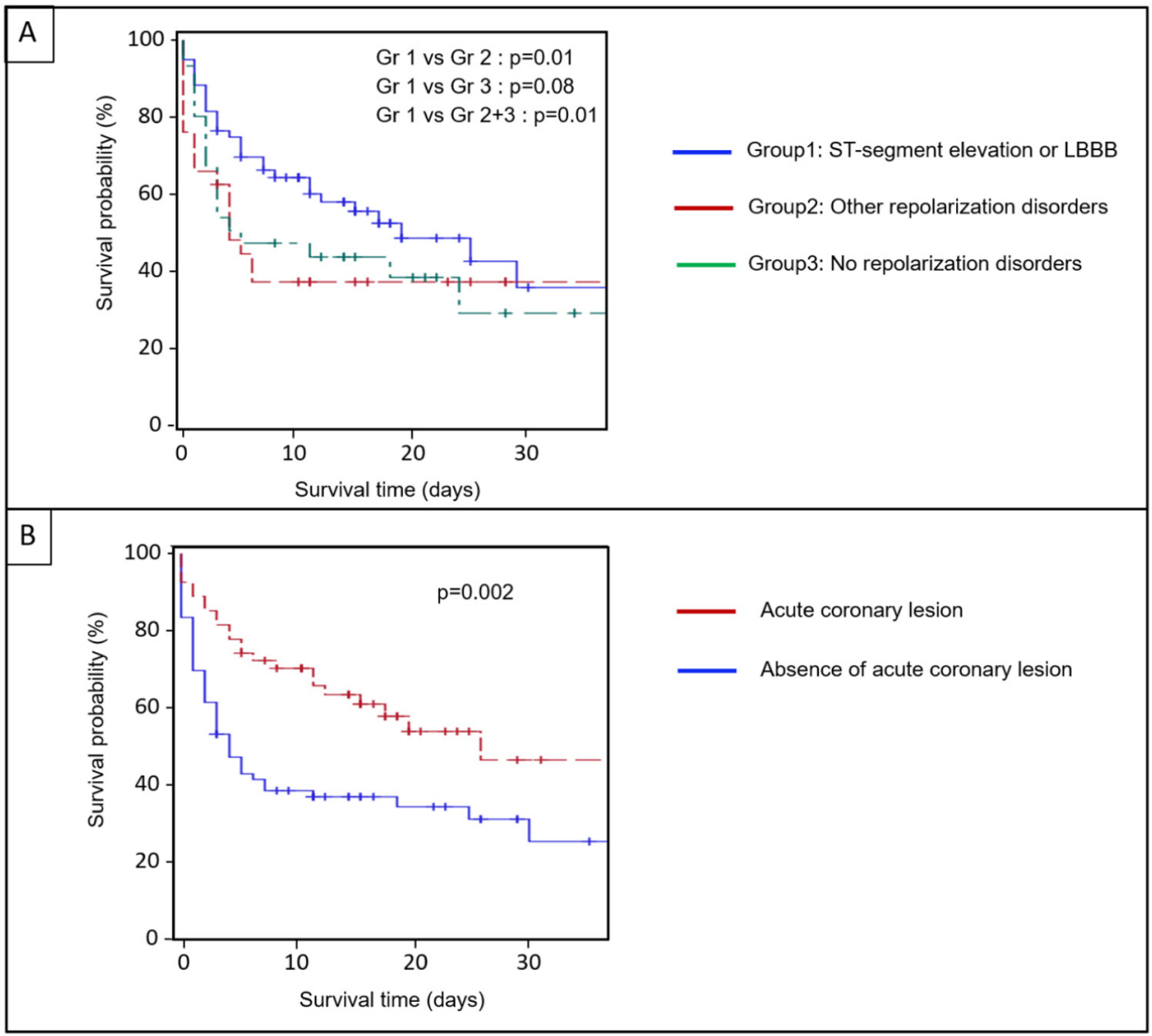

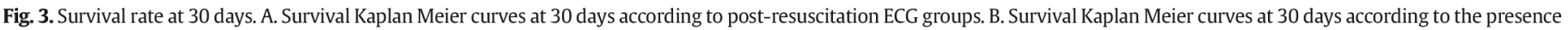
of an acute coronary lesion. LBBB means Left Bundle Branch Block. 


\section{Discussion}

Despite better knowledge and therapeutic advances, the management of OHCA is still a major challenge for physicians because of its poor prognosis. In this study, we aimed to evaluate the role of postresuscitation ECG among OHCA patients as a triage method to predict the presence of an acute coronary lesion involved in OHCA. We provided new findings that can be summarized as follows. First, persistent STE or new presumed LBBB represented more than half of the postresuscitation ECG patterns after a resuscitated OHCA. Second, the absence of any repolarization disorders was associated with a very high predictive value to exclude an acute coronary lesion requiring immediate revascularization. Third, chest pain before cardiac arrest and postresuscitation STE or new presumed LBBB was independently associated with the presence of an acute coronary lesion. Fourth, despite specific and invasive management, all-cause mortality remained very high and could be significantly reduced by immediate cardiopulmonary resuscitation by witness.

While several observational studies [9,26-29] have reported a benefit on survival of an immediate systematic CAG after OHCA, other studies did not report any reduction in mortality considering only patients without STE $[7,20,21,30]$. These conflicting results may be related to not comparable populations including intra-hospital cardiac arrests for some studies, different ECG patterns, no specific definition of acute coronary lesion, wide range of CAG timing and no standardized therapeutic management, particularly for the use of therapeutic hypothermia. In our study, the survival rate was relatively high in comparison with these observational studies. It could be explained by a selected population including only patients admitted in catheterization laboratory with a potential better prognosis, the exclusion of non-cardiac and particularly neurological causes of OHCA and by the specificities of the French health care system with MICU and trained on-board physicians. Moreover, although the independent correlates associated with a better survival in this study were a CPR initiated by a witness and a lower lactatemia, we also observed an improved survival among patients with an acute coronary lesion. The large majority of these patients were admitted with STE or LBBB. We could suggest that the better prognosis of these patients was the consequence of more frequent initial shockable rhythm and, more importantly, the result of a well-known and curable cause leading to well-organized therapeutic management. The COACT trial, which is the first randomized trial focusing on patients without STE, has provided major findings by underlining that a strategy of immediate CAG was not better than a strategy of delayed CAG with respect to overall survival at 90 days and could induce a delayed optimal intensive care. However, this trial has selected patients with a shockable rhythm and the vast majority of patients had stable coronary artery lesions [6]. Thus, the ongoing randomized EMERGE (NCT02876458) and TOMAHAWK (NCT02750462) trials should provide complementary data to define the best timing of CAG after resuscitated OHCA and the main factors to guide decision-making.

Meanwhile, it should not be forgotten that neurologic injuries remain the leading cause of death after an OHCA [6,31,32]. If a limited part of OHCA patients die from a cardiac cause, it might be difficult to improve survival by systematic CAG and revascularization of lesions other than acute coronary occlusion. Moreover, CAG is an invasive procedure with potential adverse effects such as bleeding, particularly at puncture site [22,33], renal failure, stroke, coronary dissection, rupture or occlusion. Unnecessary CAG may be associated with a potential delayed specialized and intensive care, use of unnecessary antithrombotic therapy and increased risk of stent thrombosis among instable patients [34].

As a consequence, it seems necessary to identify patients with acute coronary occlusion or instable lesion requiring urgent revascularization. In addition to the past medical history that contributes to decisionmaking of an immediate invasive strategy, chest pain before cardiac arrest and congestive heart failure symptoms are the better independent factors to predict the presence of an acute coronary lesion [5,10,35], as found in our study. However, symptom reporting is difficult and often dependent on the presence of witnesses. Thus, the decision to proceed an immediate CAG is often based on incomplete information and require additional evidence as post-resuscitation ECG. However, after performing CAG, the definition of an acute coronary lesion is challenging in the absence of acute coronary occlusion, severe coronary stenosis with TIMI flow impairment or obvious thrombus burden. An additional selective use of endo-coronary imaging should be a useful tool to guide decision-making. Indeed, using 3-vessel optical coherence tomography, Degrell et al. found that one out of four resuscitated OHCA patients without STE and without acute coronary lesion had plaque rupture, plaque erosion or thrombus [36].

In our study, the pre-specified definition of post-resuscitation ECG and acute coronary lesion allowed a specific evaluation of association between ECG patterns and acute coronary lesion and particularly acute coronary occlusion. Besides patients with STE or new presumed LBBB, only one patient had TIMI 0 flow suggesting a very limited risk of acute coronary occlusion among non-STEMI patients. More importantly, we did not observe TIMI 0/1 flow among patients without repolarization disorders whereas the potential reperfusion of an acute coronary occlusion is the main reason of performing an immediate CAG after resuscitated OHCA.

Nevertheless, some limitations of our study should be considered. First, the studied population was selected and only resuscitated OHCA patients for whom prehospital or hospital medical team decide to perform a CAG were included. As a consequence, these results and conclusions should not be extrapolated to all OHCA patients Second, follow-up was limited to 30 days but the main objective of this study was to evaluate the place of initial CAG and the proportion of acute coronary lesion depending on post-resuscitation ECG. Third, given the absence of expert or international consensus, the angiographic definition of an acute coronary lesion requiring immediate revascularization remains suggestive. However, the use of a detailed definition and an independent doubleblind reviewing may limit misdiagnosing of coronary cause of cardiac arrest, particularly for angiographically significant lesion without TIMI flow impairment or for chronic coronary occlusion. Finally, the study was under-powered to evaluate adverse events related to CAG.

In conclusion, post-resuscitation ECG appears as a useful triage method to guide the indication of immediate coronary angiography. Considering the high negative predictive value of post-resuscitation ECG to exclude acute coronary occlusion and acute coronary lesion requiring urgent revascularization after OHCA, a delayed coronary angiography is possible and safe for patients without repolarization disorders. The optimal timing to perform coronary angiography and improve its benefit/risk balance requires further analyses and randomized studies.

\section{Funding}

Investigator-based study initiative. This research did not receive any specific grant from funding agencies in the public, commercial, or notfor-profit sectors.

\section{CRediT authorship contribution statement}

Florence Leclercq: Conceptualization, Writing - review \& editing, Supervision. Clément Lonjon: Investigation, Writing - original draft. Grégory Marin: Methodology. Mariam Akodad: Investigation. François Roubille: Investigation. Jean-Christophe Macia: Investigation. Luc Cornillet: Investigation. Richard Gervasoni: Investigation. Laurent Schmutz: Investigation. Bertrand Ledermann: Investigation. Pascal Colson: Investigation. Guillaume Cayla: Conceptualization, Writing - review \& editing, Supervision. Benoit Lattuca: Conceptualization, Methodology, Writing - original draft, Writing - review \& editing. 


\section{Declaration of competing interest}

F. Leclercq has received research Grants from Boehringer Ingelheim, Edwards Lifesciences, Medtronic and consultant fees from Bayer AG, Boehringer Ingelheim and Edwards Lifesciences.

M. Akodad has received research grants from Edwards Lifescience and Medtronic.

G. Cayla has received research Grants or Consulting/Lecture Fees from Amgen, AstraZeneca, Bayer, Boehringer Ingelheim, Biotronik, Bristol-Myers Squibb, Daiichi-Sankyo, Eli-Lilly, Medtronic, MSD, Pfizer, Sanofi-Aventis.

B. Lattuca has received research grants from Biotronik, Boston Scientific, Daiichi-Sankyo, Fédération Française de Cardiologie and Institute of CardioMetabolism and Nutrition; consultant fees from DaiichiSankyo and Eli Lilly; and lecture fees from AstraZeneca, Medtronic and Novartis.

C. Lonjon, G. Marin, F. Roubille, JC. Macia, L. Cornillet, R. Gervasoni, L. Schmutz, B. Ledermann and P. Colson report no conflicts of interest in this work

\section{References}

[1] C.M. Hansen, K. Kragholm, D.A. Pearson, C. et al. Association of bystander and firstresponder intervention with survival after out-of-hospital cardiac arrest in North Carolina, 2010-2013, JAMA. 314 (2015) 255-264. doi:https://doi.org/10.1001/ jama.2015.7938.

2] S. Girotra, B.K. Nallamothu, J.A. Spertus, Y. Li, H.M. Krumholz, P.S. Chan, American Heart Association get with the guidelines-resuscitation investigators, trends in survival after in-hospital cardiac arrest, N. Engl. J. Med. 367 (2012) 1912-1920, https:// doi.org/10.1056/NEJMoa1109148.

[3] M.W. Donnino, J.C. Miller, M. Bivens, et al., A pilot study examining the severity and outcome of the post-cardiac arrest syndrome: a comparative analysis of two geographically distinct hospitals, Circulation 126 (2012) 1478-1483, https://doi.org/ 10.1161/CIRCULATIONAHA.111.067256.

[4] J. Berdowski, R.A. Berg, J.G.P. Tijssen, R.W. Koster, Global incidences of out-ofhospital cardiac arrest and survival rates: systematic review of 67 prospective studies, Resuscitation 81 (2010) 1479-1487, https://doi.org/10.1016/j.resuscitation. 2010.08.006

[5] J. Garcia-Tejada, A. Jurado-Román, J. Rodríguez, et al., Post-resuscitation electrocardiograms, acute coronary findings and in-hospital prognosis of survivors of out-ofhospital cardiac arrest, Resuscitation 85 (2014) 1245-1250, https://doi.org/10. 1016/j.resuscitation.2014.06.001.

[6] J.S. Lemkes, G.N. Janssens, N.W van der Hoeven, et al. Coronary angiography after cardiac arrest without ST-segment elevation, N. Engl. J. Med. 380 (2019) 1397-1407, https://doi.org/10.1056/NEJMoa1816897.

7] Z.A. Anyfantakis, G. Baron, P. Aubry, et al., Acute coronary angiographic findings in survivors of out-of-hospital cardiac arrest, Am. Heart J. 157 (2009) 312-318, https://doi.org/10.1016/j.ahj.2008.09.016.

[8] F. Dumas, L. White, B.A. Stubbs, A. Cariou, T.D. Rea, Long-term prognosis following resuscitation from out of hospital cardiac arrest: role of percutaneous coronary intervention and therapeutic hypothermia, J. Am. Coll. Cardiol. 60 (2012) 21-27, https://doi.org/10.1016/j.jacc.2012.03.036.

9] F. Dumas, A. Cariou, S. Manzo-Silberman, et al., Immediate percutaneous coronary intervention is associated with better survival after out-of-hospital cardiac arrest: insights from the PROCAT (Parisian Region Out of hospital Cardiac Arrest) registry, Circ. Cardiovasc. Interv. 3 (2010) 200-207, https://doi.org/10.1161/ CIRCINTERVENTIONS.109.913665.

[10] C.M. Spaulding, L.M. Joly, A. Rosenberg, et al., Immediate coronary angiography in survivors of out-of-hospital cardiac arrest, N. Engl. J. Med. 336 (1997) 1629-1633, https://doi.org/10.1056/NEJM199706053362302.

[11] S.G. Priori, C. Blomström-Lundqvist, A. Mazzanti, et al., 2015 ESC guidelines for the management of patients with ventricular arrhythmias and the prevention of sudden cardiac death, Eur. Heart J. 36 (2015) 2793-2867, https://doi.org/10.1093/eurheartj/ ehv316.

[12] F.-J. Neumann, M. Sousa-Uva, A. Ahlsson, et al., ESC scientific document group, 2018 ESC/EACTS guidelines on myocardial revascularization, Eur. Heart J. 40 (2) (2019 Jan 7) 87-165, https://doi.org/10.1093/eurheartj/ehy394.

[13] B. Ibanez, S. James, S. Agewall, et al., 2017 ESC guidelines for the management of acute myocardial infarction in patients presenting with ST-segment elevation: the task force for the management of acute myocardial infarction in patients presenting with ST-segment elevation of the European Society of Cardiology (ESC). Eur. Heart J 39 (2) (2018 Jan 7) 119-177, https://doi.org/10.1093/eurheartj/ehx393.

[14] Z.J. Zheng, J.B. Croft, W.H. Giles, G.A. Mensah, Sudden cardiac death in the United States, 1989 to 1998 , Circulation. 104 (2001) 2158-2163.

[15] D.P. Zipes, H.J.J. Wellens, Sudden cardiac death, Circulation 98 (1998) 2334-2351, https://doi.org/10.1161/01.CIR.98.21.2334.

[16] J.P. Pell, J.M. Sirel, A.K. Marsden, I. Ford, N.L. Walker, S.M. Cobbe, Presentation, management, and outcome of out of hospital cardiopulmonary arrest: comparison by underlying aetiology, Heart 89 (2003) 839-842, https://doi.org/10.1136/heart.89. 8.839.

[17] M.J. Davies, Anatomic features in victims of sudden coronary death. Coronary artery pathology. Circulation. 85 (1992) I19-24.

[18] N. Patel, N.J. Patel, C.J. Macon, et al., Trends and outcomes of coronary angiography and percutaneous coronary intervention after out-of-hospital cardiac arrest associated with ventricular fibrillation or pulseless ventricular tachycardia, JAMA Cardiol. 1 (2016) 890-899, https://doi.org/10.1001/jamacardio.2016.2860.

[19] F. Dumas, W. Bougouin, G. Geri, et al., Emergency percutaneous coronary intervention in post-cardiac arrest patients without ST-segment elevation pattern: insight from the PROCAT II registry, JACC Cardiovasc. Interv. 9 (2016) 1011-1018, https:// doi.org/10.1016/j.jcin.2016.02.001.

[20] I.I. Staudacher, C. den Uil, L. Jewbali, et al., Timing of coronary angiography in survivors of out-of-hospital cardiac arrest without obvious extracardiac causes, Resuscitation 123 (2018) 98-104, https://doi.org/10.1016/j.resuscitation.2017.11.046.

[21] J. Dankiewicz, N. Nielsen, M. Annborn, et al., Survival in patients without acute ST elevation after cardiac arrest and association with early coronary angiography: a post hoc analysis from the TTM trial, Intensive Care Med. 41 (2015) 856-864, https://doi.org/10.1007/s00134-015-3735-z.

[22] R.D. Hollenbeck, J.A. McPherson, M.R. Mooney, et al., Early cardiac catheterization is associated with improved survival in comatose survivors of cardiac arrest without STEMI, Resuscitation 85 (2014) 88-95, https://doi.org/10.1016/j.resuscitation. 2013.07.027.

[23] K. Thygesen, J.S. Alpert, H.D. White, et al., ESC Committee for practice guidelines (CPG), third universal definition of myocardial infarction, Eur. Heart J. 33 (2012) 2551-2567, https://doi.org/10.1093/eurheartj/ehs184.

[24] I.B. Menown, G. Mackenzie, A.A. Adgey, Optimizing the initial 12-lead electrocardiographic diagnosis of acute myocardial infarction, Eur. Heart J. 21 (2000) 275-283, https://doi.org/10.1053/euhj.1999.1748.

[25] J.H. Chesebro, G. Knatterud, R. Roberts, et al. Thrombolysis in myocardial infarction (TIMI) trial, Phase I: a comparison between intravenous tissue plasminogen activator and intravenous streptokinase. Clinical findings through hospital discharge, Circulation. 76 (1987) 142-154

[26] G. Geri, F. Dumas, W. Bougouin, et al. Immediate percutaneous coronary intervention is associated with improved short- and long-term survival after out-ofhospital cardiac arrest, Circ. Cardiovasc. Interv. 8 (2015). doi:https://doi.org/10 1161/CIRCINTERVENTIONS.114.002303.

[27] A. Vyas, P.S. Chan, P. Cram, B.K. Nallamothu, B. McNally, S. Girotra, Early coronary angiography and survival after out-of-hospital cardiac arrest, Circ. Cardiovasc. Interv. 8 (2015). doi:https://doi.org/10.1161/CIRCINTERVENTIONS.114.002321.

[28] K.B. Kern, K. Lotun, N. Patel, et al., INTCAR-cardiology registry, outcomes of comatose cardiac arrest survivors with and without ST-segment elevation myocardial infarction: importance of coronary angiography, JACC Cardiovasc. Interv. 8 (2015) 1031-1040, https://doi.org/10.1016/j.jcin.2015.02.021.

[29] M.S. Khan, S.M.M. Shah. A. Mubashir, et al., Early coronary angiography in patients resuscitated from out of hospital cardiac arrest without ST-segment elevation: a systematic review and meta-analysis, Resuscitation 121 (2017) 127-134, https://doi. org/10.1016/j.resuscitation.2017.10.019.

[30] J. Bro-Jeppesen, J. Kjaergaard, M. Wanscher, et al., Emergency coronary angiography in comatose cardiac arrest patients: do real-life experiences support the guidelines? Eur. Heart J. Acute Cardiovasc. Care 1 (2012) 291-301, https://doi.org/10.1177/ 2048872612465588.

[31] S. Laver, C. Farrow, D. Turner, J. Nolan, Mode of death after admission to an intensive care unit following cardiac arrest, Intensive Care Med. 30 (2004) 2126-2128, https://doi.org/10.1007/s00134-004-2425-z.

[32] N. Nielsen, J. Wetterslev, T. Cronberg, et al., TTM trial investigators, targeted temperature management at $33^{\circ} \mathrm{C}$ versus $36^{\circ} \mathrm{C}$ after cardiac arrest, N. Engl. J. Med. 369 (2013) 2197-2206, https://doi.org/10.1056/NEJMoa1310519.

[33] G. Casella, V. Carinci, P. Cavallo, et al., Combining therapeutic hypothermia and emergent coronary angiography in out-of-hospital cardiac arrest survivors: optima post-arrest care for the best patient, Eur. Heart J. Acute Cardiovasc. Care 4 (2015) 579-588, https://doi.org/10.1177/2048872614564080.

[34] J. Joffre, O. Varenne, W. Bougouin, J. Rosencher, J.-P. Mira, A. Cariou, Stent thrombosis: an increased adverse event after angioplasty following resuscitated cardiac arrest, Resuscitation 85 (2014) 769-773, https://doi.org/10.1016/j.resuscitation. 2014.02.013.

[35] S.W. Waldo, L. Chang, J.B. Strom, C. O'Brien, E. Pomerantsev, R.W. Yeh, Predicting the presence of an acute coronary lesion among patients resuscitated from cardiac arrest, Circ. Cardiovasc. Interv. 8 (2015). doi:https://doi.org/10.1161/ CIRCINTERVENTIONS.114.002198.

[36] P. Degrell, F. Picard, N. Combaret, et al., Coronary atherothrombosis in cardiac arrest survivors without ST-segment elevation on ECG, Resuscitation. 139 (2019) 189-191, https://doi.org/10.1016/j.resuscitation.2019.01.046. 